\title{
Pain Neuroscience Education for Children with Functional Abdominal Pain Disorders: A Randomized Comparative Pilot Study
}

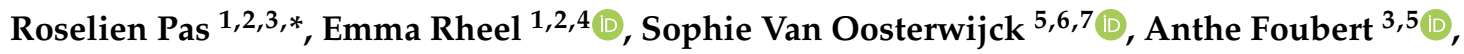 \\ Robby De Pauw ${ }^{6}\left(\mathbb{D}\right.$, Laurence Leysen ${ }^{1,2}\left(\mathbb{D}\right.$, Ann Roete ${ }^{8}$, Jo Nijs ${ }^{1,2,9}{ }^{(D}$, Mira Meeus $1,3,6,+(\mathbb{D})$ \\ and Kelly Ickmans $1,2,7,9,+\mathbb{D}$ \\ 1 Pain in Motion Research Group (PAIN), Department of Physiotherapy, Human Physiology and Anatomy, \\ Faculty of Physical Education and Physiotherapy, Vrije Universiteit Brussel, Laarbeeklaan 103, \\ 1090 Brussels, Belgium; emma.rheel@vub.be (E.R.); laurence.leysen@vub.be (L.L.); jo.nijs@vub.be (J.N.); \\ mira.meeus@uantwerpen.be (M.M.); kelly.ickmans@vub.be (K.I.) \\ 2 Department of Physiotherapy, Human Physiology and Anatomy (KIMA), Faculty of Physical \\ Education \& Physiotherapy, Vrije Universiteit Brussel (VUB), Laarbeeklaan 103, 1090 Brussels, Belgium \\ 3 Department of Rehabilitation Sciences and Physiotherapy, Faculty of Medicine and Health Sciences, \\ University of Antwerp (UA), D.S.022, 2610 Wilrijk, Belgium; Anthe.Foubert@uantwerpen.be \\ 4 Department of Experimental-Clinical and Health Psychology, Ghent University, Henri Dunantlaan 2, \\ 9000 Ghent, Belgium \\ 5 Pain in Motion International Research Group; Sophie.VanOosterwijck@UGent.be \\ 6 Department of Rehabilitation Sciences, Faculty of Medicine and Health Sciences, Ghent University, \\ Campus UZ, Corneel Heymanslaan 10, 9000 Ghent, Belgium; Robby.DePauw@UGent.be \\ 7 Research Foundation-Flanders (FWO), 1000 Brussels, Belgium \\ 8 Antwerp University Hospital, department of Pediatrics, 2610 Wilrijk, Belgium; ann.roete@uza.be \\ 9 Department of Physical Medicine and Physiotherapy, University Hospital Brussels, 1090 Brussels, Belgium \\ * Correspondence: Roselien.pas@vub.be; Tel.: +32-(0)2-477-45-29 \\ + Both authors contributed equally to the manuscript as last author.
}

Received: 17 April 2020; Accepted: 3 June 2020; Published: 9 June 2020

\begin{abstract}
This article explores the effectiveness of a newly developed Pain Neuroscience Education program for children (PNE4Kids) with functional abdominal pain disorder (FAPD). Children (6-12 years) with FAPD were randomly assigned to 1$)$ the experimental group $(n=14)$, participating in one hypnotherapy session (i.e., usual care) and one additional PNE4Kids session, or 2) the control group $(n=14)$, participating in two hypnotherapy sessions. Parental pain catastrophizing, the child's functional disability (parental-proxy), pain-related fear (parent-proxy) and pain intensity, were assessed at baseline and one and three weeks after each therapy session. Pressure algometry and a conditioned pain modulation paradigm were performed at baseline and three weeks after completion of the last therapy session. Parents from both the experimental as well as the control group showed significantly less parental pain catastrophizing $(p<0.01)$. Children showed significantly less functional disability $(p<0.05)$, pain-related fear $(p<0.01)$ and local pressure pain sensitivity $(p<0.05)$ at short-term follow-up (three weeks after last intervention) in both groups. No significant $(p>0.05)$ between-group differences were found. Hypnotherapy combined with PNE4Kids did not result in better clinical outcomes compared to hypnotherapy alone. Study limitations include the application of one single PNE4Kids session and the short follow-up time.
\end{abstract}

Keywords: hypnotherapy; catastrophizing; disability; fear; hyperalgesia 


\section{Introduction}

Functional Abdominal Pain Disorders (FAPDs) are prevalent in up to $13.5 \%$ of children and adolescents and have a significant impact on their health-related quality of life as well as their physical, academic and social activities and exposes children at a higher risk to the development of chronic pain and depressive symptoms later in life [1-4]. Children with FAPD might develop negative cognitions when they do not understand the origin of the pain they experience and should therefore be educated about their condition $[5,6]$. Current pain management in adolescents and in children with persistent pain often includes an educational component [7]. Nevertheless, this psychoeducation is focused on psychological aspects related to pain and on pain management strategies rather than on an explanation of the pain science, including underlying biological mechanisms of pain [8]. Indeed, it might be counterintuitive to patients (and parents) to advise them to adopt certain behaviors (i.e., resuming physical, social, and school activities) without decreasing the threat value of pain first.

Pain Neuroscience Education (PNE) therefore (1) includes a thorough explanation of pain science; (2) aims to reassure patients (and parents) by decreasing the threat value of pain; and (3) includes the integral role of psychosocial and physical factors in precipitating and maintaining pain. Therefore, PNE may prepare and prime children with chronic pain and their parents for biopsychosocial treatments [7,9-11]. PNE has been used in various adult chronic pain populations and has been shown to be effective in changing pain beliefs (i.e., pain catastrophizing) and pain coping strategies, improving disability, pain intensity, and health status $[12,13]$.

The limited studies investigating PNE(-based) interventions in the pediatric chronic pain context, only include (healthy) adolescents and their parents $[10,14,15]$. Drawing upon available evidence in adult and adolescent samples, it is expected that PNE provided to children (6-12 years) and their parents will lead to improved pain-related outcomes in both the child and the parents. Involving the parents of children with FAPD in PNE sessions will facilitate an increased understanding of biopsychosocial factors that influence pain and might indirectly improve the child's pain-related outcomes. Parents who catastrophize about their child's pain show an encouragement of child pain behaviors [16], and promote more child anxiety and pain-related fear [17]. In addition, parents' catastrophizing about their child's pain has been found to predict child- and parent-reported functional disability and school attendance [18]. By including parents in therapy, they will learn how they can support their child in managing their symptoms.

To date, one of the most commonly used treatment modalities in children with FAPD is hypnotherapy. In general, hypnotherapy consists of exercises to induce general relaxation, as well as exercises to control abdominal pain and gut functions, all of which are aimed at increasing the child's well-being. Hypnotherapy was proven to be effective in reducing pain in the short term in children with FAPD [19]. Given the innovative feature of PNE4Kids [7,20], ethical considerations have led us to use it in combination with the most commonly used therapy in Belgium, rather than investigating PNE4Kids as a stand-alone treatment. The present study examined whether children with FAPD and their parents receiving PNE4Kids in addition to standard care (i.e., hypnotherapy) would help improve parental pain catastrophizing and child's functional disability (parental proxy) when compared to a group receiving only standard care. Secondly, we hypothesize that children with FAPD and their parents will show an improvement in pain intensity, pain-related fear, primary and secondary pressure hyperalgesia, and endogenous pain inhibition.

\section{Experimental Section}

\subsection{Setting and Participants}

Ethical approval for this 2-armed randomized controlled pilot study was obtained from the Ethics Committee of the University of Antwerp/Antwerp University Hospital and was part of another study examining endogenous pain modulation in children with FAPD [21]. The full study, including this part, was registered at clinicaltrials.gov (NCT02880332) in August 2016. Participants included boys 
and girls aged between 6 and 12 years who were presented at the pediatric department of the Antwerp University Hospital for abdominal pain. Other inclusion criteria were (1) the child had a history of pain for at least 3 months; (2) a diagnosis with FAPD by the pediatric abdominal pain specialist, based on the Rome III criteria [22]; and (3) the child was able to speak and read Dutch fluently (in an age-dependent manner). Exclusion criteria included children who (1) previously participated in one or more PNE session(s); (2) were diagnosed with a concomitant chronic disease (e.g., diabetes mellitus); (3) were born prematurely [23]; (4) previously participated in a study assessing experimental pain; or (5) presented with contra-indications to complete the experimental pain measures [24].

At the beginning of the study, children from the age of 12 and their parents were asked to sign the informed consent form. Participants were asked to refrain from performing heavy physical activity and from the intake of analgesics on the day of the pain testing procedure.

\subsection{Study Flow}

Participants were randomized by (R.P.) to either the control or the experimental group by using a computerized random number generator for concealment. Both assessors (A.F. and S.V.O) were blind after the assignment. The procedure of the study and the CONSORT diagram including participant flow are presented in Figure 1.

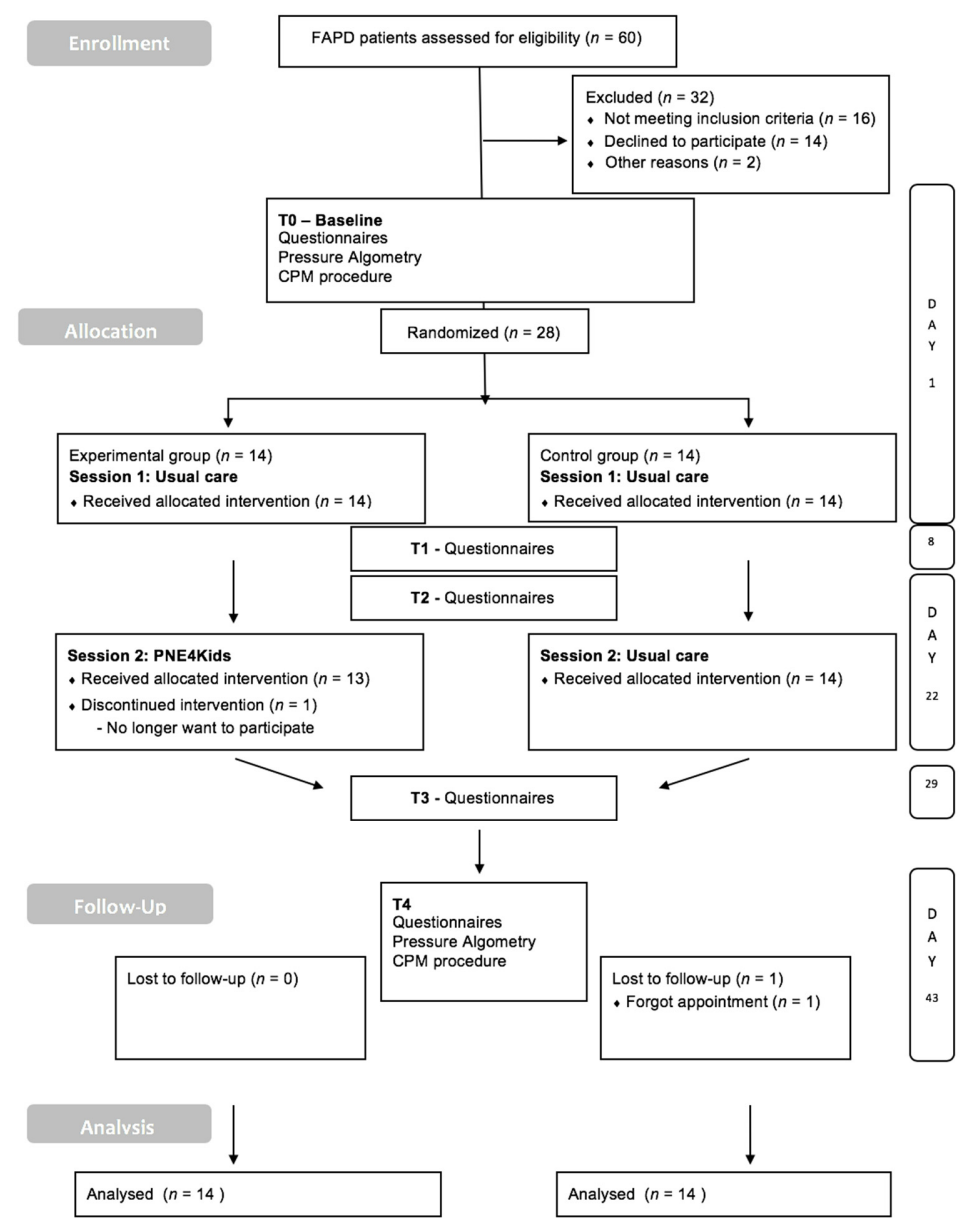

Figure 1. CONSORT Flow diagram.

\subsection{Questionnaires}

Parent: The Dutch Parent version of the Pain Catastrophizing Scale (PCS-P) was assessed, describing the different thoughts and feelings that parents may experience about their child's pain [18]. Total scores range from $0-52$, with higher scores indicating more pain catastrophizing about their 
child's pain. In addition, three subscales can be calculated for (1) rumination; (2) magnification; and (3) helplessness. This scale has been reported to be a valid measurement tool [18]. The Dutch version of the Functional Disability Inventory-parent proxy (FDI-P) was used to measure difficulties in physical and psychosocial functioning during the past two weeks [25]. Total scores ranged from 0 to 60 , with higher scores indicating greater functional disability. This tool has shown good validity and reliability measures in children with chronic abdominal pain [25]. The Dutch Fear of Pain Questionnaire-parent report (FOPQ-P) is a parent proxy report to assess a child's pain-related fear and avoidance behavior. Total scores range from 0-92, with a higher score representing more fear [26]. This questionnaire has shown good psychometric properties [26].

Children: The Faces Pain Scale-Revised (FPS-R) was used to assess the child's average abdominal pain intensity from the previous week. This scale consists of six faces, presented horizontally, which relate to a numeric value from 0 ("no pain") to 10 ("the worst imaginable pain"). This scale has been recommended for research purposes based on its utility and psychometric features in children between 4 and 12 years [27,28]; it has also been used in cases of chronic pain [29].

\subsection{Experimental Pain Measurements}

The procedure of experimental pain testing has been described in detail previously [21]. Briefly, children completed two laboratory pain tasks in absence of their parents (pressure algometry and conditioned pain modulation by using the cold pressor task; more information is available in Appendix A).

\subsection{Interventions}

Control group: Children in the control group received two sessions of hypnotherapy (usual care). The first $+/-1 \mathrm{~h}$ interactive session was used to have the participant become familiar with the therapist (a nurse with specific expertise in hypnotherapy) and hypnosis techniques. These techniques included visualizations of a normal working gut (e.g., using metaphors adapted to the child's interests, such as a car running at a normal speed). No fixed hypnotic scripts were used, the hypnosis technique was modified based on the child's interests and by using feedback from the child. The second session included of a revision of the content given in the first session and additional breathing and relaxation exercises. Parents attended each therapy session, however they did not interact.

Experimental group: Children within the experimental group participated in one hypnotherapy session (usual care) plus Pain Neuroscience Education for children (PNE4Kids). The first session had the same content as the first session of the control group (described above). The second session, however, was different and consisted of $\mathrm{a}+/-1 \mathrm{~h}$ one-on-one interactive educational session about the neurophysiology of pain using a board game. The PNE4Kids program was developed based on the book "Explain pain" by Butler and Moseley and includes an explanation and reassurance on the cause of pain, a brief summary of relevant pain mechanisms, and the integral role of psychosocial and physical factors in precipitating and maintaining pain [30]. The "Explain pain" material, which was initially written for adults, was adapted to the cognitive comprehensible stage of children and to the specific pain population (i.e., FAPD). More detailed information on these adaptations and on the exact content of the PNE4kids program can be read elsewhere [11] and is available via http://www.paininmotion.be/PNE4Kids. Parents attended each therapy session, however they did not interact.

\subsection{Statistical Analyses}

All data were analyzed using IBM SPSS Statistics for Macintosh, Version 24.0 Armonk, NY, USA IBM Corp. Analysis was done by intention to treat. Demographic variables were compared between groups by a Chi-square test for categorical variables and a Mann-Whitney $U$ test for continuous variables with the significance level set at $5 \%$. Based on these analyses, none of the demographic variables showed significant between-group differences $(p>0.05)$. To assess between-group difference in response to treatment, a random-intercept linear mixed models analysis was applied. A likelihood ratio 
test was implemented to assess the significance of the included variables. The model included treatment (experimental or control intervention), time (5 measurement points; T0-T4), and treatment $\times$ time as fixed effects together with a random intercept for each participant. Only significant interaction effects (i.e., treatment $\times$ time) were kept in the model. Primary analysis was performed on the two primary outcome measures; PSC-P (total score) and the FDI-P. Bonferroni-corrected significance levels were set at alpha $=0.025$ to correct for multiple comparison (family-wise error rate (FWER) $<0.05$ ). Besides, the estimated marginal mean for each time point was calculated based on the final model. Effect sizes of the mean time differences were calculated as the Cohen's $\mathrm{d}$.

\section{Results}

Patient enrollment took place between February 2017 and October 2018. A total of 28 children with FAPD were included in the study and received either two sessions of hypnotherapy (usual care) (control intervention; $n=14$ ) or either a combination of one session of hypnotherapy usual care and one session of PNE4Kids (experimental intervention; $n=14$ ) (Figure 1). Both intervention groups lost one participant; one participant did no longer want to participate (T2), while another participant forgot his/her follow-up appointment (T4). The demographic and baseline characteristics of the participants are presented in Table 1.

Table 1. Demographic and Baseline Characteristics of the participants.

\begin{tabular}{|c|c|}
\hline Demographic & Participants No. (\%) \\
\hline \multicolumn{2}{|l|}{ Child sex } \\
\hline \multicolumn{2}{|l|}{ Experimental group } \\
\hline Female & $9(64.3)$ \\
\hline Male & $5(35.7)$ \\
\hline \multicolumn{2}{|l|}{ Control group } \\
\hline Female & $9(64.3)$ \\
\hline Male & $5(35.7)$ \\
\hline \multicolumn{2}{|l|}{ Parental sex } \\
\hline \multicolumn{2}{|l|}{ Experimental group } \\
\hline Female & $11(78.6)$ \\
\hline Male & $3(21.4)$ \\
\hline \multicolumn{2}{|l|}{ Control group } \\
\hline Female & $11(78.6)$ \\
\hline Male & $3(21.4)$ \\
\hline \multicolumn{2}{|l|}{ Parental history of chronic Pain } \\
\hline \multicolumn{2}{|l|}{ Experimental group } \\
\hline Yes & $5(35.7)$ \\
\hline No & $9(64.3)$ \\
\hline \multicolumn{2}{|l|}{ Control group } \\
\hline Yes & $4(28.6)$ \\
\hline No & $10(71.7)$ \\
\hline \multicolumn{2}{|l|}{ Race child } \\
\hline \multicolumn{2}{|l|}{ Experimental group } \\
\hline Caucasian & $13(92.9)$ \\
\hline Non-Caucasian & $1(7.1)$ \\
\hline \multicolumn{2}{|l|}{ Control group } \\
\hline Caucasian & $13(92.9)$ \\
\hline Non-Caucasian & $1(7.1)$ \\
\hline \multicolumn{2}{|l|}{ Parental educational level } \\
\hline \multicolumn{2}{|l|}{ Experimental group } \\
\hline Low ( $\leq$ high school level education) & $5(35.7)$ \\
\hline Middle (Bachelor's degree or equivalent) & $6(42.9)$ \\
\hline High $(\geq$ Master's degree $)$ & $3(21.4)$ \\
\hline \multicolumn{2}{|l|}{ Control group } \\
\hline Low ( $\leq$ high school level education) & $5(35.7)$ \\
\hline Middle (Bachelor's degree or equivalent) & $5(35.7)$ \\
\hline High $(\geq$ Master's degree) & $4(28.6)$ \\
\hline
\end{tabular}


Table 1. Cont.

\begin{tabular}{|c|c|c|}
\hline Demographic & Mean (SD) & Median (IQR) (Range) \\
\hline \multicolumn{3}{|l|}{ Child age, years } \\
\hline Experimental group & $9.21(1.53)$ & 9.00 (3.00) (7.00-12.00) \\
\hline Control group & $8.71(1.73)$ & $9.00(3.25)(6.00-11.00)$ \\
\hline \multicolumn{3}{|l|}{ Parental age, years } \\
\hline Experimental group & $39.57(4.18)$ & 39.00 (5.25) (35.00-49.00) \\
\hline Control group & $37.57(4.65)$ & $38.00(7.75)(29.00-44.00)$ \\
\hline \multicolumn{3}{|l|}{ Pain duration, months } \\
\hline Experimental group & $19.71(12.68)$ & 17.00 (15.75) (4.00-48.00) \\
\hline Control group & $28.36(21.40)$ & $24.00(39.25)(3.00-70.00)$ \\
\hline \multicolumn{3}{|l|}{ Pain episodes, $\mathrm{n} /$ month } \\
\hline Experimental group & $21.29(8.73)$ & 23.50 (14.25) (3.00-31.00) \\
\hline Control group & $20.64(14.73)$ & 16.50 (17.75) (3.00-60.00) \\
\hline Baseline & Mean (SD) & Median (IQR) [Range] \\
\hline \multicolumn{3}{|l|}{ FDI-P score } \\
\hline Experimental group & $11.00(11.60)$ & 6.50 (16.25) (0.00-43.00) \\
\hline Control group & $9.21(8.54)$ & $7.00(6.25)(2.00-34.00)$ \\
\hline \multicolumn{3}{|l|}{ FPS-R score } \\
\hline Experimental group & $3.71(2.20)$ & 4.00 (2.50) (0.00-8.00) \\
\hline Control group & $5.14(2.80)$ & $5.00(4.50)(0.00-10.00)$ \\
\hline \multicolumn{3}{|l|}{ FOPQ-P score } \\
\hline Experimental group & $41.50(14.85)$ & $37.50(23.75)(24.00-73.00)$ \\
\hline Control group & $31.71(9.23)$ & $34(14.25)(12.00-42.00)$ \\
\hline \multicolumn{3}{|l|}{ PCS-P score } \\
\hline Experimental group & $19.79(9.54)$ & 19.00 (12.25) (3.00-41.00) \\
\hline Control group & $22.29(10.25)$ & $23.50(9.50)(2.00-40.00)$ \\
\hline \multicolumn{3}{|l|}{ PPT Trapezius, $\mathrm{kg} / \mathrm{cm}^{2}$} \\
\hline Experimental group & $1.41(0.85)$ & $1.17(0.61)(0.73-4.00)$ \\
\hline Control group & $1.15(0.43)$ & $1.02(0.70)(0.68-2.05)$ \\
\hline \multicolumn{3}{|l|}{ PPT Umbilicus, $\mathrm{kg} / \mathrm{cm}^{2}$} \\
\hline Experimental group & $1.04(0.52)$ & 1.05 (0.77) (0.13-2.10) \\
\hline Control group & $0.95(0.32)$ & $0.84(0.36)(0.50-1.61)$ \\
\hline \multicolumn{3}{|l|}{ PPT Tibia, kg/ $\mathrm{cm}^{2}$} \\
\hline Experimental group & $2.61(1.12)$ & 2.59 (1.44) (1.19-5.00) \\
\hline Control group & $2.47(1.05)$ & $2.36(1.39)(1.08-4.50)$ \\
\hline \multicolumn{3}{|l|}{ CPM, absolute difference } \\
\hline Experimental group & $-0.01(0.59)$ & $-0.07(0.51)(-0.63-1.76)$ \\
\hline Control group & $-0.28(0.37)$ & $-0.19(1.10)(-1.08-0.30)$ \\
\hline \multicolumn{3}{|l|}{$\mathrm{CPM}, \%$ change } \\
\hline Experimental group & $-7.90(26.45)$ & $-6.75(40.17)(-50.00-44.12)$ \\
\hline Control group & $-32.64(39.88)$ & $-22.54(73.06)(-105.19-24.49)$ \\
\hline
\end{tabular}

Abbreviations: IQR: Interquartile range; SD: Standard deviation; PCS-P: Pain Catastrophizing Scale for Parents (score: 0-52); FDI-P: Functional Disability Inventory-Parent proxy (score: 0-60); FOPQ-P: Fear of Pain Questionnaire-Parent version (score: 0-92); FPS-R: Faces Pain Scale-Revised (range 0-10); PPT: Pain Pressure Threshold; CPM: Conditioned Pain Modulation; CPT: Cold Pressor Task; Calculations: CPM absolute difference $=($ PPT Trapezius-PPT Trapezius during CPM $)$; CPM Percentage change $=((($ PPT Trapezius-PPT Trapezius during CPM $) /$ PPT Trapezius during $\left.(\mathrm{CPM})^{*} 100\right)$.

\subsection{Primary Outcomes}

The PCS-P and FDI-P did not show a significant interaction effect, although a significant main effect of time for both primary outcome measurements were found (Table 2, Figure 2).

For the total PCS-P significant improvements (medium-to-high effect sizes) from baseline were found at three weeks after session one (T2) (estimated marginal mean difference (diff.), 4.74; 95\% CI, -0.31 to 6.03 ), one week after therapy completion (T3) (estimated marginal mean diff., 5.37, 95\% CI, 2.16 to 8.51 ), and three weeks after therapy completion (T4) (estimated marginal mean diff., $6.07,95 \% \mathrm{CI}$, 2.93 to 9.21$)$. 
Table 2. Main group, time, and interaction effect for the primary and secondary outcomes.

\begin{tabular}{|c|c|c|c|c|c|c|}
\hline \multirow[t]{2}{*}{ Model } & \multicolumn{3}{|c|}{ Group } & \multicolumn{2}{|c|}{ Time } & Group $\times$ Time \\
\hline & AIC & $F(d f)$ & $P$-Value & $F(d f)$ & $P$-Value & F (df) $\quad P$-Value \\
\hline \multicolumn{7}{|c|}{ Primary outcomes } \\
\hline PCS-P & 825.5 & $0.292(1)$ & 0.593 & $9.831(4)$ & $<0.001$ & NA \\
\hline FDI-P & 882.1 & $0.959(1)$ & 0.337 & $3.272(4)$ & 0.014 & NA \\
\hline \multicolumn{7}{|c|}{ Secondary outcomes } \\
\hline POFQ-P & 928.0 & $1.620(1)$ & 0.214 & $4.527(4)$ & 0.002 & NA \\
\hline FPS-R & 628.2 & $2.622(1)$ & 0.117 & $2.536(4)$ & 0.045 & NA \\
\hline PCS-P (Rum.) & 626.0 & $0.069(1)$ & 0.791 & $6.800(4)$ & $<0.001$ & NA \\
\hline PCS-P (Magn.) & 463.8 & $0.445(1)$ & 0.511 & $2.440(4)$ & 0.052 & NA \\
\hline PCS-P (Help.) & 650.3 & $0.439(1)$ & 0.514 & $8.947(4)$ & $<0.001$ & NA \\
\hline PPT Trapezius & 92.8 & $1.356(1)$ & 0.255 & $0.074(1)$ & 0.788 & NA \\
\hline PPT Umbilicus & 54.7 & $0.207(1)$ & 0.653 & $5.745(1)$ & 0.024 & NA \\
\hline PPT Tibia & 146.0 & $0.524(1)$ & 0.476 & $4.226(1)$ & 0.050 & NA \\
\hline CPM, Abs Diff. & 72.788 & $1.928(1)$ & 0.177 & $3.176(1)$ & 0.087 & NA \\
\hline CPM, \% change & 492.291 & $3.343(1)$ & 0.080 & $1.063(1)$ & 0.314 & NA \\
\hline
\end{tabular}

Abbreviations: PCS-P: Pain Catastrophizing Scale for Parents (score: 0-52); FDI-P: Functional Disability Inventory-Parent proxy (score: 0-60); FOPQ-P: Fear of Pain Questionnaire-Parent version (score: 0-92); FPS-R: Faces Pain Scale-Revised (range 0-10); PPT: Pain Pressure Threshold; CPM: Conditioned Pain Modulation; CPT: Cold Pressor Task; AIC: Akaike information criterion; (df): Degrees of freedom; NA: Not applicable; Calculations: CPM absolute difference $=($ PPT Trapezius-PPT Trapezius during CPM $) ;$ CPM Percentage change $=((($ PPT Trapezius-PPT Trapezius during (PM)/PPT Trapezius during (PM)*100).
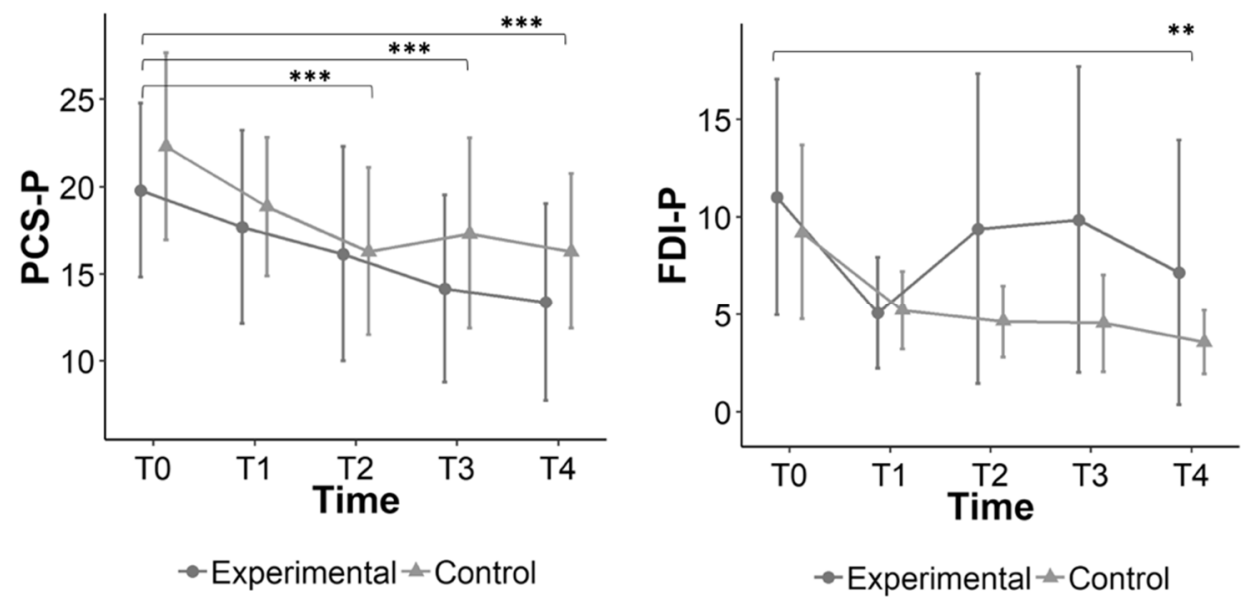

Figure 2. Graphs showing the mean difference over time for the primary outcomes with significant main time effect, $*: p<0.05 ;^{* *}: p<0.01{ }^{* * *}: p<0.00$. Abbreviations: PCS-P: Pain Catastrophizing Scale for Parents (score: 0-52); FDI-P: Functional Disability Inventory-Parent proxy (score: 0-60);

For the FDI-P, significant improvements (medium effect size) were found from baseline to three weeks after therapy completion (T4) (estimated marginal mean diff., 5.05; 95\% CI, 0.86 to 9.24).

The FOPQ-P showed improvements (medium effect size) from baseline to one week after therapy completion (T3) (estimated marginal mean diff., 5.52; 95\% CI, 0.75 to 10.30) and three weeks after therapy completion (T4) (estimated marginal mean diff., 5.73; 95\% CI, 1.02 to 10.45).

Table 3 shows the mean and standard error for the interaction effects of the primary outcome measures. 
Table 3. Mean and standard error for the interaction effects of the primary outcome measures.

\begin{tabular}{ccc}
\hline $\begin{array}{c}\text { Primary Outcomes and Their } \\
\text { Measurement in Time (T) }\end{array}$ & $\begin{array}{c}\text { Experimental Group } \\
\text { Mean (S.E.) }\end{array}$ & $\begin{array}{c}\text { Control Group } \\
\text { Mean (S.E) }\end{array}$ \\
\hline PCS-P & $19.786(2.554)$ & $22.286(2.554)$ \\
0 & $17.541(2.602)$ & $18.857(2.554)$ \\
1 & $16.385(2.580)$ & $16.286(2.554)$ \\
2 & $14.385(2.580)$ & $17.021(2.576)$ \\
3 & $13.616(2.580)$ & $16.286(2.554)$ \\
4 & & \\
FDI-P & $11.000(2.593)$ & $9.214(2.593)$ \\
0 & $7.540(2.676)$ & $5.214(2.593)$ \\
1 & $8.806(2.637)$ & $4.643(2.593)$ \\
2 & $9.268(2.637)$ & $4.751(2.631)$ \\
3 & $6.576(2.637)$ & $3.571(2.593)$ \\
4 & &
\end{tabular}

\subsection{Secondary Outcomes}

\subsubsection{Questionnaires}

The PCS-P subscale rumination showed significant improvements (medium-to-high effect sizes) from baseline to three weeks after session one (T2) (estimated marginal mean diff., $1.85 ; 95 \%$ CI, 0.38 to 3.32), one week after therapy completion (T3) (estimated marginal mean diff., $1.93,95 \%$ CI, 0.44 to 3.41), and three weeks after therapy completion (T4) (estimated marginal mean diff., $2.26 ; 95 \% \mathrm{CI}$, 0.79 to 3.73). For the PCS-P helplessness subscale, significant improvements (medium-to-high effect sizes) were found from baseline to one week after session one (T2) (estimated marginal mean diff., $1.86 ; 95 \%$ CI, 0.22 to 3.49 ), three weeks after session one (T2) (estimated marginal mean diff., $2.32 ; 95 \%$ CI, 0.70 to 3.95), one week after therapy completion (T3) (estimated marginal mean diff., $2.74 ; 95 \%$ CI, 1.10 to 4.39 ), and three weeks after therapy completion (T4) (estimated marginal mean diff., 3.03; $95 \%$ CI, 1.40 to 4.65$)$.

The FOPQ-P showed improvements (medium effect size) from baseline to one week after therapy completion (T3) (estimated marginal mean diff., 5.52; 95\% CI, 0.75 to 10.30) and three weeks after therapy completion (T4) (estimated marginal mean diff., 5.73; 95\% CI, 1.02 to 10.45).

The FPS-R did not show a significant interaction effect, nor a significant main effect of time.

\subsubsection{Experimental Pain Measurements}

None of the experimental pain measurements (locale and remote pressure hyperalgesia + conditioned pain modulation) showed a significant interaction effect (Table 2, Figure 2). However, for the PPT at the umbilicus test site a significant improvement (small effect size) was found at three weeks after therapy completion (T4) (estimated marginal mean diff., $-0.15 ; 95 \%$ CI, -0.29 to 0.02 ).

\section{Discussion}

This study is the first to examine the combined effect of hypnotherapy and PNE4Kids in children with FAPD on parental pain catastrophizing and the parental estimation of the child's functional disability. This exploratory randomized controlled trial was designed to gather valuable information regarding the effectiveness of PNE4Kids, to steer further research and clinics.

One session of PNE4Kids combined with one session of hypnotherapy (usual care) did not result in significantly better outcomes when compared to the control group receiving two sessions of usual care (i.e., hypnotherapy). Still, both intervention groups appeared to improve in the short-term (T4; at three weeks following the last intervention) regarding parental pain catastrophizing and functional disability. 
A variety of reasons might explain why no significant differences between the experimental and control group were found.

Both hypnotherapy and PNE4Kids are hypothesized to improve pain through similar mechanisms. The aim of PNE4Kids is to help children with FAPD and their parents in their understanding of the pain onset and progress of the disorder. The reconceptualization of pain might induce changes in insight and thereby changes in pain cognitions [11]. Hypnotherapy on the other hand, may bring about cognitive changes throughout by directly influencing cognitions, which helps to improve symptoms, or through influencing pain and gut functioning, which also leads to a change in cognition [31]. Hence, both treatment interventions might have induced comparable results, making it hard to find significant differences between both treatment groups.

Secondly, the sequence of the PNE4kids delivery (i.e., after the first usual care session) might have reduced its effects. Given the hypothesis that the reconceptualization of pain via PNE increases treatment responses, PNE is preferably given prior to additional exercises or cognitive and behavioral interventions [32]. In this study however, one PNE4Kids session was provided in addition to one usual care session to ensure the therapist giving the first intervention session was blind. Similar to pain management in adults with chronic pain, PNE should be used to enhance a time-contingent, cognition-targeted approach in the performance of (physical) activities [33], which are often impaired in children with FAPD [34]. Evidence of the isolated effect of PNE is available after one-on-one sessions in adults with chronic pain [35-37]. Although, the dosage of PNE sessions highly vary between studies, from one single session (of a duration of $3 \mathrm{~h}$ ) in the study of Moseley et al. (2004) to four sessions in the study of Werner et al. (2016) Therefore it might be important to conduct follow-up educational sessions including a recapitulation of previous information in children. In addition, it might be useful to investigate the influence of other PNE delivery methods, including group sessions or blended learning [38].

Third, previous studies providing evidence for the effect of PNE in healthy children [10] and the context of pediatric pain has mainly focused on attitudes and knowledge of pain physiology, instead of pain-related outcomes such as physical functioning, pain intensity and pain-related fear [14,15]. Despite its frequent use in the evaluation of functional disability in the context of functional abdominal pain $[39,40]$, it should be noted that the FDI was initially developed to assess perceived difficulty in a variety of activities that are relevant to children and adolescents, rather than to assess limitations specific to a particular pediatric condition such as FAPDs [25]. When analyzing the descriptive statistics of the separate items of the FDI, the two most reported disabling items were getting asleep and staying asleep (item 25; 89\%) and eating regular meals (item 5; 64\%). On the contrary, watching television (item 11) and staying awake all day (item 6) had the lowest ratings by parent report (respectively $7 \%$ and $18 \%$ ). Including other outcomes, such as children's knowledge of pain physiology, might have been valuable in assessing the additional use of PNE4Kids. To date, however, no valid and reliable assessment tool exists to assess children's (6-12 years old) knowledge of pain.

There are some limitations to report regarding this study. First, we did not include a statistical analysis plan in the registered protocol of this study. Secondly, the use of parent-proxy questionnaires might have led to an under-estimation of children's pain-related functioning, making it more difficult to detect treatment effects. Indeed, several items of the FDI refer to academic or social activities that parents may not directly observe. Including children from the age of six hampered the possibility of using direct measurements, since no questionnaire assessing pain catastrophizing was validated for our targeted age group. Third, the therapist providing the usual care was involved in both treatment arms, and although care was taken to ascertain that the PNE therapist was not involved in the usual care, it cannot be excluded that the therapist providing the usual care did not include aspects of PNE in their treatment. Study strengths include the randomized controlled design using balanced treatment arms, well-defined experimental treatment, a priori training of the therapists, clinical trial registration, and the use of blinded assessments. 


\section{Conclusions}

Within this study, no significant differences were found between hypnotherapy (i.e., usual care including biomedical education) and hypnotherapy with PNE4Kids. Since additional PNE4Kids is not found to be worse either, further studies might examine treatment satisfaction and cost effectiveness among other features. Future studies should also investigate whether a combined approach of PNE4Kids applied as a preparatory therapy modality followed by a cognition-targeted active approach is able to improve pain-related outcomes in children with FAPD.

Author Contributions: Conceptualization, R.P.; K.I.; M.M.; methodology, M.M.; K.I.; R.P.; formal analysis, R.P.; S.V.O.; A.F.; R.D.P.; investigation, R.P.; S.V.O.; A.F.; A.R.; resources, A.R.; writing-original draft preparation, R.P.; writing-review and editing, J.N.; E.R.; M.M.; K.I.; L.L.; visualization, R.D.P.; E.R.; L.L.; supervision, M.M.; K.I.; project administration, K.I.; funding acquisition, J.N.; K.I.; M.M. All authors have read and agreed to the published version of the manuscript.

Funding: Grant support for as R. and Dra. Rheel E. was provided by a Chair funded by the Berekuyl Academy/European College for Decongestive Lymphatic Therapy, the Netherlands and awarded to the Vrije Universiteit Brussel, Belgium. Sophie Van Oosterwijck is a researcher supported by a research project grant from the Research Foundation-Flanders (FWO) (grant number G0B3718N). Kelly Ickmans is a postdoctoral research fellow partly funded by the Research Foundation-Flanders (FWO).

Acknowledgments: The authors would like to thank Els Van de Vijver for her help in recruiting patients.

Conflicts of Interest: Jo Nijs, Roselien Pas and Kelly Ickmans have co-authored a Dutch book for clinicians on pain neuroscience education, but the royalties for that book are collected by the Vrije Universiteit Brussel and not them personally. Besides that, the authors have no conflict of interest relevant to this article to disclose. The funders had no role in the design of the study; in the collection, analyses, or interpretation of data; in the writing of the manuscript; or in the decision to publish the results.

\section{Appendix A}

Pressure hyperalgesia was assessed with the Force Dial model FDK 40 algometer (Wagner Instruments, Greenwich, UK) by administering pressure via a small flat rubber disc with a surface area of $1 \mathrm{~cm}$ to the umbilical (local site) [41], and the trapezial [41] and tibial regions (both remote sites) [42] at the dominant side. The children were instructed to say "stop" at the moment when the sensation of the mechanical stimulus applied to their body changed from pressure to pain. A total of three measurements (pressure pain threshold (PPT) measurements) were performed at each testing site, with $30 \mathrm{~s}$ between each test. The mean of the last two PPTs per test site was calculated and used for further analyses.

To assess efficiency of endogenous pain inhibition, the conditioned pain modulation (CPM) paradigm was performed by combining pressure algometry and the cold pressor task. The water circulator apparatus cooled water to $12{ }^{\circ} \mathrm{C} \pm 1{ }^{\circ} \mathrm{C}$ and had a continuous flow and circulation rate. The child was instructed to immerse its non-dominant hand in the circulating water. Following an initial immersion of $20 \mathrm{~s}$, the PPT at the trapezial region was reassessed three times, with an inter-stimulus duration of $30 \mathrm{~s}$. Immediately after the last PPT measurement, the child was instructed to withdraw its hand out of the water and was offered a towel to dry and warm the hand. Again, the mean of the latter two PPTs was calculated. The outcome measure for CPM was obtained by the following formula: ((PPT preCPM-PPT postCPM)/PPT pre-CPM)*100.

\section{Appendix B}

As this paper was somewhat exploratory in nature, we deviated slightly from our registered protocol, and we report those deviations here in the spirit of transparency.

We originally planned to explore the influence of the intervention on the primary outcome parental pain catastrophizing (PCS-P), but decided to add a second primary outcome measure focusing on the child, this being a pain-related disability. Based on the results of a recent Cochrane literature review which included 18 randomized controlled trials, it became clear that studies investigating pain-related disability following hypnotherapy in children with functional abdominal pain were lacking [43]. 
We planned to report on the child's pain related to fear using parent-reported (The Dutch Fear of Pain Questionnaire-parent report) [26] as well as child-reported measurements (The Kuttner Anxiety Scale). The reason why we did not report the results on the child-reported Kuttner Anxiety Scale was mainly because we could not find any other study reporting the reliability and validity measures of this scale.

\section{References}

1. Hyams, J.S.; Di Lorenzo, C.; Saps, M.; Shulman, R.J.; Staiano, A.; Van Tilburg, M. Childhood functional gastrointestinal disorders: Child/adolescent. Gastroenterology 2016, 150, 1456-1468.e2. [CrossRef] [PubMed]

2. Korterink, J.J.; Diederen, K.; Benninga, M.A.; Tabbers, M.M. Epidemiology of pediatric functional abdominal pain disorders: A meta-analysis. PLoS ONE 2015, 10, e0126982. [CrossRef]

3. Walker, L.S.; Dengler-Crish, C.M.; Rippel, S.; Bruehl, S. Functional abdominal pain in childhood and adolescence increases risk for chronic pain in adulthood. Pain 2010, 150, 568-572. [CrossRef] [PubMed]

4. Youssef, N.N.; Atienza, K.; Langseder, A.L.; Strauss, R.S. Chronic abdominal pain and depressive symptoms: Analysis of the national longitudinal study of adolescent health. Clin. Gastroenterol. Hepatol. 2008, 6, 329-332. [CrossRef] [PubMed]

5. Meldrum, M.L.; Tsao, J.C.; Zeltzer, L.K. “I can't be what I want to be": Children's narratives of chronic pain experiences and treatment outcomes. Pain Med. 2009, 10, 1018-1034. [CrossRef] [PubMed]

6. Jackson, T.; Pope, L.; Nagasaka, T.; Fritch, A.; Iezzi, T.; Chen, H. The impact of threatening information about pain on coping and pain tolerance. Br. J. Health Psychol. 2005, 10, 441-451. [CrossRef]

7. Robins, H.; Perron, V.; Heathcote, L.C.; Simons, L.E. Pain Neuroscience Education: State of the Art and Application in Pediatrics. Children 2016, 3, 43. [CrossRef]

8. Moseley, G.L.; Butler, D.S. Fifteen Years of Explaining Pain: The Past, Present, and Future. J. Pain 2015, 16, 807-813. [CrossRef]

9. Malfliet, A.; Leysen, L.; Pas, R.; Kuppens, K.; Nijs, J.; Van Wilgen, P.; Huysmans, E.; Goudman, L.; Ickmans, K. Modern pain neuroscience in clinical practice: Applied to post-cancer, paediatric and sports-related pain. Braz. J. Phys. Ther. 2017, 21, 225-232. [CrossRef]

10. Louw, A.; Podalak, J.; Zimney, K.; Schmidt, S.; Puentedura, E.J. Can pain beliefs change in middle school students? A study of the effectiveness of pain neuroscience education. Physiother. Theory Pract. 2018, 34, 542-550. [CrossRef]

11. Pas, R.; Meeus, M.; Malfliet, A.; Baert, I.; Oosterwijck, S.V.; Leysen, L.; Nijs, J.; Ickmans, K. Development and feasibility testing of a Pain Neuroscience Education program for children with chronic pain: Treatment protocol. Braz. J. Phys. Ther. 2018, 22, 248-253. [CrossRef] [PubMed]

12. Watson, J.A.; Ryan, C.G.; Cooper, L.; Ellington, D.; Whittle, R.; Lavender, M.; Dixon, J.; Atkinson, G.; Cooper, K.; Martin, D.J. Pain Neuroscience Education for Adults With Chronic Musculoskeletal Pain: A Mixed-Methods Systematic Review and Meta-Analysis. J. Pain 2019, 20, 1140.e1-1140.e22. [CrossRef] [PubMed]

13. Louw, A.; Zimney, K.; Puentedura, E.J.; Diener, I. The efficacy of pain neuroscience education on musculoskeletal pain: A systematic review of the literature. Physiother. Theory Pract. 2016, 32, 332-355. [CrossRef]

14. Neto, M.; Andias, R.; Silva, A.G. Pain Neuroscience Education and Exercise for Neck Pain:A Focus Group Study on Adolescents' Views. Pediatr. Phys. Ther. 2018, 30, 196-201. [CrossRef]

15. Reid, K.; Hartling, L.; Ali, S.; Le, A.; Norris, A.; Scott, S.D. Development and Usability Evaluation of an Art and Narrative-Based Knowledge Translation Tool for Parents With a Child With Pediatric Chronic Pain: Multi-Method Study. J. Med. Internet Res. 2017, 19, e412. [CrossRef]

16. Lynch-Jordan, A.; Kashikar-Zuck, S.; Szabova, A.; Goldschneider, K. The Interplay of Parent and Adolescent Catastrophizing and Its Impact on Adolescents' Pain, Functioning, and Pain Behavior. Clin. J. Pain 2013, 29, 681-688. [CrossRef]

17. Chow, E.T.; Otis, J.D.; Simons, L.E. The Longitudinal Impact of Parent Distress and Behavior on Functional Outcomes Among Youth With Chronic Pain. J. Pain 2016, 17, 729-738. [CrossRef] 
18. Goubert, L.; Eccleston, C.; Vervoort, T.; Jordan, A.; Crombez, G. Parental catastrophizing about their child's pain. The parent version of the Pain Catastrophizing Scale (PCS-P): A preliminary validation. Pain 2006, 123, 254-263. [CrossRef]

19. Abbott, R.A.; Martin, A.E.; Newlove-Delgado, T.V.; Bethel, A.; Whear, R.S.; Thompson Coon, J.; Logan, S. Recurrent Abdominal Pain in Children: Summary Evidence From 3 Systematic Reviews of Treatment Effectiveness. J. Pediatr. Gastroenterol. Nutr. 2018, 67, 23-33. [CrossRef]

20. Heathcote, L.C.; Allen, J.M.; Gunn, K.M.; Fox, S.; Harvie, D.S.; Olver, I.; Skinner, I.W.; Smith, A.G.; Stanton, T.R.; Whitford, H.S.; et al. Pain Education for Adolescents and Young Adults Living Beyond Cancer: An Interdisciplinary Meeting Report. J. Adolesc. Young Adult Oncol. 2019, 8, 529-533. [CrossRef]

21. Pas, R.; Rheel, E.; Van Oosterwijck, S.; Leysen, L.; Van De Vijver, E.; Nijs, J.; Ickmans, K.; Meeus, M. Endogenous pain modulation in children with functional abdominal pain disorders. Pain 2019, 160, 1883-1890. [CrossRef]

22. Drossman, D.A. The functional gastrointestinal disorders and the Rome III process. Gastroenterology 2006, 130, 1377-1390. [CrossRef]

23. Goffaux, P.; Lafrenaye, S.; Morin, M.; Patural, H.; Demers, G.; Marchand, S. Preterm births: Can neonatal pain alter the development of endogenous gating systems? European J. Pain 2008, 12, 945-951. [CrossRef]

24. von Baeyer, C.L.; Piira, T.; Chambers, C.T.; Trapanotto, M.; Zeltzer, L.K. Guidelines for the cold pressor task as an experimental pain stimulus for use with children. J. Pain 2005, 6, 218-227. [CrossRef]

25. Claar, R.; Walker, L. Functional assessment of pediatric pain patients: Psychometric properties of the functional disability inventory. Pain 2006, 121, 77-84. [CrossRef]

26. Simons L, S.C.; Carpino, E.; Logan, D.; Berde, C. The Fear of Pain Questionnaire (FOPQ): Assessment of pain-related fear among children and adolescents with chronic pain. J. Pain 2011, 12, 677-686. [CrossRef]

27. Stinson, J.N.; Kavanagh, T.; Yamada, J.; Gill, N.; Stevens, B. Systematic review of the psychometric properties, interpretability and feasibility of self-report pain intensity measures for use in clinical trials in children and adolescents. Pain 2006, 125, 143-157. [CrossRef]

28. Tomlinson, D.; von Baeyer, C.L.; Stinson, J.N.; Sung, L. A systematic review of faces scales for the self-report of pain intensity in children. Pediatrics 2010, 126, e1168-e1198. [CrossRef]

29. Lee, R.R.; Rashid, A.; Ghio, D.; Thomson, W.; Cordingley, L. Chronic Pain Assessments in Children and Adolescents: A Systematic Literature Review of the Selection, Administration, Interpretation, and Reporting of Unidimensional Pain Intensity Scales. Pain Res. Manag. 2017, 2017, 7603758. [CrossRef]

30. Butler, D.; Moseley, G. Explain Pain, 1st ed.; NOI Group Publishing: Adelaide, Australia, 2003.

31. Vlieger, A.M.; Rutten, J.M.; Govers, A.M.; Frankenhuis, C.; Benninga, M.A. Long-term follow-up of gut-directed hypnotherapy vs. standard care in children with functional abdominal pain or irritable bowel syndrome. Am. J. Gastroenterol. 2012, 107, 627-631. [CrossRef]

32. Nijs, J.; Paul van Wilgen, C.; Van Oosterwijck, J.; van Ittersum, M.; Meeus, M. How to explain central sensitization to patients with 'unexplained' chronic musculoskeletal pain: Practice guidelines. Man. Ther. 2011, 16, 413-418. [CrossRef] [PubMed]

33. Malfliet, A.; Kregel, J.; Coppieters, I.; De Pauw, R.; Meeus, M.; Roussel, N.; Cagnie, B.; Danneels, L.; Nijs, J. Effect of Pain Neuroscience Education Combined With Cognition-Targeted Motor Control Training on Chronic Spinal Pain: A Randomized Clinical Trial. JAMA Neurol. 2018, 75, 808-817. [CrossRef] [PubMed]

34. van der Veek, S.M.; Derkx; de Haan, E.; Benninga, M.; Boer, F. Abdominal Pain in Dutch Schoolchildren: Relations With Physical and Psychological Comorbid Complaints in Children and Their Parents. Gastroenterology 2010, 51, 481-487. [CrossRef] [PubMed]

35. Louw, A.; Diener, I.; Butler, D.S.; Puentedura, E.J. The effect of neuroscience education on pain, disability, anxiety, and stress in chronic musculoskeletal pain. Arch. Phys. Med. Rehabil. 2011, 92, 2041-2056. [CrossRef]

36. Werner, E.L.; Storheim, K.; Lochting, I.; Wisloff, T.; Grotle, M. Cognitive Patient Education for Low Back Pain in Primary Care: A Cluster Randomized Controlled Trial and Cost-Effectiveness Analysis. Spine (Phila Pa 1976) 2016, 41, 455-462. [CrossRef]

37. Moseley, G.L.; Nicholas, M.K.; Hodges, P.W. A randomized controlled trial of intensive neurophysiology education in chronic low back pain. Clin. J. Pain 2004, 20, 324-330. [CrossRef]

38. Malfliet, A.; Kregel, J.; Meeus, M.; Roussel, N.; Danneels, L.; Cagnie, B.; Dolphens, M.J.N. Blended-Learning Pain Neuroscience Education for People With Chronic Spinal Pain: Randomized Controlled Multicenter Trial. Phys. Ther. 2018, 98, 357-368. [CrossRef] 
39. Wendland, M.; Jackson, Y.; Stokes, L.D. Functional disability in paediatric patients with recurrent abdominal pain. Child. Care Health Dev. 2010, 36, 516-523. [CrossRef]

40. Cunningham, N.R.; Lynch-Jordan, A.; Barnett, K.; Peugh, J.; Sil, S.; Goldschneider, K.; Kashikar-Zuck, S. Child pain catastrophizing mediates the relation between parent responses to pain and disability in youth with functional abdominal pain. J. Pediatr. Gastroenterol. Nutr. 2014, 59, 732-738. [CrossRef]

41. Duarte, M.a.; Goulart, E.M.; Penna, F.J. Pressure pain threshold in children with recurrent abdominal pain. J. Pediatr. Gastroenterol. Nutr. 2000, 31, 280-285. [CrossRef]

42. Linari-Melfi, M.; Cantarero-Villanueva, I.; Fernández-Lao, C.; Fernández-De-Las-Peñas, C.; Guisado-Barrilao, R.; Arroyo-Morales, M. Analysis of deep tissue hypersensitivity to pressure pain in professional pianists with insidious mechanical neck pain. BMC Musculoskelet. Disord. 2011, 12, 268. [CrossRef]

43. Abbott, R.A.; Martin, A.E.; Newlove-Delgado, T.V.; Bethel, A.; Thompson-Coon, J.; Whear, R.; Logan, S. Psychosocial interventions for recurrent abdominal pain in childhood. Cochrane Database Syst. Rev. 2017, 1, CD010971. [CrossRef] [PubMed]

(C) 2020 by the authors. Licensee MDPI, Basel, Switzerland. This article is an open access article distributed under the terms and conditions of the Creative Commons Attribution (CC BY) license (http://creativecommons.org/licenses/by/4.0/). 